\title{
An Analysis of Sequential Auctions for Common and Private Value Objects
}

\author{
Shaheen S. Fatima ${ }^{1} \quad$ Michael Wooldridge $^{1} \quad$ Nicholas R. Jennings $^{2}$ \\ ${ }^{1}$ Department of Computer Science, \\ University of Liverpool, Liverpool L69 7ZF, U.K. \\ \{S.S.Fatima, M.J.Wooldridge\}@csc.liv.ac.uk \\ ${ }^{2}$ School of Electronics and Computer Science, \\ University of Southampton, Southampton SO17 1BJ, U.K. \\ nrj@ecs.soton.ac.uk
}

\begin{abstract}
Sequential auctions are an important mechanism for buying/selling multiple objects. Existing work has studied sequential auctions for objects that are exclusively either common value or private value. However, in many real-world cases an object has both features. Also, in such cases, the common value depends on how much each bidder values the object. Moreover, a bidder generally does not know the true common value (since it may not know how much the other bidders value it). Given this, our objective is to study settings that have both common and private value elements by treating each bidder's information about the common value as uncertain. Each object is modelled with two signals: one for its common value and the other for its private value. The auctions are conducted using English auction rules. For this model, we first determine equilibrium bidding strategies for each auction in a sequence. On the basis of this equilibrium, we find the expected selling price and the winner's expected profit for each auction. We then show that even if the common and private values of objects are distributed identically across all objects, the selling price and the winner's profit are not the same for all of them. We show that, in accordance with Ashenfelter's experimental results [1], the selling price for our model can decline in later auctions. Finally, we show that the selling prices and the winner's expected profits in an agent-based setting can differ from those in an all-human setting.
\end{abstract}

\section{Introduction}

Market-based mechanisms such as auctions are now being widely studied as a means of buying/selling resources in multiagent systems. This uptake is occurring because auctions are both simple and have a number of desirable properties (typically the most important of which are their ability to generate high revenues to the seller and to allocate resources efficiently) $[19,4,21]$. Now, in many cases the number of objects to be auctioned is greater than one. There are two types of auctions that are used for multiple objects: combinatorial auctions [18] and sequential auctions $[6,3,11]$. The former are used when the objects for sale are available at the same time, while the latter (which are the main focus of this paper) are used when the objects become available at different points in time. In the sequential case, the auctions are conducted at different times, 
therefore a bidder may participate in more than one auction. In such a scenario, it has been shown that although there is only one object being auctioned at a time, the bidding behaviour for any individual auction strongly depends on the auctions that are yet to be conducted $[6,3]$. For example, consider sequential auctions for oil exploration rights. In this scenario, the price an oil company will pay for a given area is affected not only by the area that is available in the current round, but also by the areas that will become available in subsequent rounds of leasing. Thus, it would be foolish for a bidder to spend all the money set aside for exploration on the first round of leasing, if potentially even more favourable sites are likely to be auctioned off subsequently.

Against this background, a key problem in the area is to study the strategic behaviour of bidders in each individual auction. To date, considerable research effort has been devoted to this problem, but an important shortcoming of existing work on sequential auctions is that it focuses on objects that are either exclusively private value (different bidders value the same object differently) or exclusively common value (an object is worth the same to all bidders) $[15,22,17,10,6]$. Furthermore, some of this work also makes the complete information assumption [17, 2]. However, most auctions are neither exclusively private nor common value, but involve an element of both [12]. Again, consider the above example of auctioning oil-drilling rights. This is, in general, treated as a common value auction. But private value differences may arise, for example, when a superior technology enables one firm to exploit the rights better than others. Also, in such cases, the common value (which is the same for all the bidders) depends on how much each bidder values the object. Moreover, generally speaking an individual bidder does not know the true common value, since it is unlikely to know how much the other bidders value it. On the other hand, the private value of a bidder is independent of the other bidders' private values.

Given this, our objective is to study sequential auctions for the general case where there are both common and private value elements. We do this by modelling each object with a two-dimensional signal: one for its common value and the other for its private value component. Each bidder's information about the common value is uncertain. Also, each bidder needs at most one object. The auctions are conducted using English auction rules. For this model, we first determine equilibrium bidding strategies for each auction in a sequence. On the basis of this equilibrium, we find the expected selling price and the winner's expected profit for each auction. We show that even if the common and private values are distributed identically across all objects, the selling price and the winner's profit are not the same for all of them ${ }^{1}$. Specifically, we consider an example scenario and show that in accordance with Ashenfelter's empirical result [1], the selling price for our model can decline in later auctions. Finally, we show that the selling prices and the winner's expected profits in an agent-based setting differ from those in an all human setting. This happens because these two settings differ in terms of

\footnotetext{
${ }^{1}$ This study is important because Ashenfelter [1] showed a declining price anomaly: in realworld sequential auctions mean sale prices for identical objects decline in later auctions. In contrast, for objects that are exclusively common/private value, the theoretical results of Milgrom and Weber [20,14], and McAfee and Vincent [13] show a completely opposite effect. Our objective is therefore to show that, for our model, the selling prices can decline in later auctions.
} 
competition - an agent-based setting results in relatively higher competition (i.e., more bidders) [16].

Our paper therefore makes three important contributions to the state of the art in multi-object auctions. First, we determine equilibrium bidding strategies for sequential auctions that involve both common and private value elements. Second, we show that, in accordance with Ashenfelter's experimental results [1], the selling price can decline in later auctions. Third, we show that the selling prices and the winner's expected payoffs in a series of auctions in an agent-based setting can differ from those in an all-human setting.

The remainder of the paper is organised as follows. Section 2 describes the auction setting. Section 3 determines equilibrium bidding strategies. In Section 4, we present an example auction scenario to illustrate a decline in the selling price of later auctions. We also show that competition can affect the expected selling prices and the winner's expected profits. Section 5 provides a discussion of how our result relates with existing work on sequential auctions. Section 6 concludes. Appendix A to C provide proofs of theorems.

\section{The sequential auctions model}

Single object auctions that have both private and common value elements have been studied in [8]. We therefore adopt this basic model and extend it to cover the multiple objects case. Before doing so, however, we give an overview of the basic model.

Single object. A single object auction is modelled in [8] as follows. There are $n \geq 3$ risk neutral bidders. The common value $\left(V_{1}\right)$ of the object to the $n$ bidders is equal, but initially the bidders do not know this value. However, each bidder receives a signal that gives an estimate of this common value. Bidder $i=1, \ldots, n$ draws an estimate $\left(v_{i 1}\right)$ of the object's true value $\left(V_{1}\right)$ from the probability distribution function $Q(v)$ with support $\left[v_{L}, v_{H}\right]$. Although different bidders may have different estimates, the true value $\left(V_{1}\right)$ is the same for all bidders and is modelled as the average of the bidders' signals: $V_{1}=\frac{1}{n} \sum_{i=1}^{n} v_{i 1}$. Furthermore, each bidder has a cost which is different for different bidders and this cost is its private value. For $i=1, \ldots, n$, let $c_{i 1}$ denote bidder $i$ 's signal for this private value which is drawn from the distribution function $G(c)$ with support $\left[c_{L}, c_{H}\right]$ where $c_{L} \geq 0$ and $v_{L} \geq c_{H}$. Cost and value signals are independently and identically distributed across bidders. Henceforth, we will use the term value to refer to common value and cost to refer to private value.

If bidder $i$ wins the object and pays $b$, it gets a utility of $V_{1}-c_{i 1}-b$, where $V_{1}-c_{i 1}$ is $i$ 's surplus. Each bidder bids so as to maximize its utility. Note that bidder $i$ receives two signals ( $v_{i 1}$ and $c_{i 1}$ ) but its bid has to be a single number. Hence, in order to determine their bids, bidders need to combine the two signals into a summary statistic. This is done as follows. For $i$, a one-dimensional summary signal, called $i$ 's surplus ${ }^{2}$, is defined as:

$$
S_{i 1}=v_{i 1} / n-c_{i 1}
$$

\footnotetext{
${ }^{2}$ Note that $i$ 's true surplus is $V_{1}-c_{i 1}$ which is equal to $v_{i 1} / n-c_{i 1}+\sum_{j \neq i} v_{j 1} / n$. But since $v_{i 1} / n-c_{i 1}$ depends on $i$ 's signals while $\sum_{j \neq i} v_{j 1} / n$ depends on the other bidders' signals, the term ' $i$ 's surplus' is also used to mean $v_{i 1} / n-c_{i 1}$.
} 
which allows $i$ 's optimal bids to be determined in terms of $S_{i 1}$ (see [8] for more details about the problems with two signals and why a one-dimensional surplus is required). In order to rank bidders from low to high valuations, $Q(v)$ and $G(c)$ are assumed to be $\log$ concave $^{3}$. Under this assumption, the conditional expectations $E(v \mid S=x)$ and $E(v \mid S \leq x)$ are non-decreasing in $x$. Furthermore, $E(c \mid S=x)$ and $E(c \mid S \leq x)$ are non-increasing in $x$. In other words, the bidders can be ranked from low to high values on the basis of their surplus. We now extend this model to $m>1$ objects.

Multiple objects ${ }^{4}$. For each of the $m>1$ objects, the bidders' values are independently and identically distributed and so are their costs. There are $m$ distribution functions for the common values, one for each object. Likewise, there are $m$ distribution functions for the costs, one for each object. For $j=1, \ldots, m$, let $Q_{j}: R_{+} \rightarrow[0,1]$ denote the distribution function for the value of the $j$ th object and $G_{j}: R_{+} \rightarrow[0,1]$ that for its cost. Thus, each bidder receives its value signal for the $j$ th object from $Q_{j}$ and its cost signal from $G_{j}$.

Furthermore, each bidder receives the cost and value signals for an auction just before that auction begins. The signals for the $j$ th object are received only after the $(j-1)$ previous auctions have been conducted. Consequently, although the bidders know the distribution functions from which the signals are drawn, they do not know the actual signals for the $j$ th object until the previous $(j-1)$ auctions are over.

The $m$ objects are sold one after another in $m$ auctions that are conducted using English auction rules. Furthermore, each bidder can win at most one object. The winner for the $j$ th object cannot participate in the remaining $m-j$ auctions. Thus, if $n$ agents participate in the first auction, the number of agents for the $j$ th auction is $(n-j+1)$. For objects $j=1, \ldots, m$ and bidders $i=1, \ldots, n$, let $v_{i j}$ and $c_{i j}$ denote the common and private values respectively. The true common value of the $j$ th object (denoted $V_{j}$ ) is:

$$
V_{j}=\frac{1}{n-j+1} \sum_{i=1}^{n-j+1} v_{i j}
$$

For objects $j=1, \ldots, m$ and bidders $i=1, \ldots, n$, let $S_{i j}=v_{i j} / n-c_{i j}$ denote $i$ 's surplus for object $j$.

Note that the values/costs for our model are not correlated. Such correlations occur across objects, if for a bidder (say $i$ ) the value/cost of object $j=2, \ldots, m$ can be determined on the basis of $i$ 's value/cost signal for the first object. However, in many cases such a direct relation between the objects may not exist. Hence, we focus on the case where different objects have different distribution functions. Furthermore, although each bidder knows the distribution functions from which the values/costs are drawn before the first auction begins, it receives its signals for an object only just before

\footnotetext{
${ }^{3} \log$ concavity means that the natural $\log$ of the densities is concave. This restriction is met by many commonly used densities like uniform, normal, chi-square, and exponential, and it ensures that optimal bids are increasing in surplus. Again see [8] for more details.

${ }^{4}$ Our model for multiple objects is a generalisation of [3]. While [3] studies sequential auctions for two private value objects, we study sequential auctions for $m \geq 2$ objects that have both private and common values.
} 
the auction for that object begins. In the following section, we determine equilibrium bidding strategies for this model.

\section{Equilibrium bidding strategies}

The $m$ objects are auctioned in $m$ separate English auctions that are conducted sequentially. The English auction rules are as follows. The auctioneer continuously raises the price, and bidders publicly reveal when they withdraw from the auction. Bidders who drop out from an auction are not allowed to re-enter that auction. A bidder's strategy for the $j$ th (for $j=1, \ldots, m-1$ ) auctions depends on how much profit it expects to get from the $(m-j)$ auctions yet to be conducted. However, since there are only $m$ objects there are no more auctions after the $m$ th one. Thus, a bidder's strategic behaviour during the last auction is the same as that for a single object English auction. Equilibrium bidding strategies for a single object of the type described in Section 2 have been obtained in [8]. We therefore briefly summarize these strategies and then determine equilibrium for our $m$ objects case.

Single object. For a single object with value $V_{1}$, the equilibrium obtained in [8] is as follows. A bidder's strategy is described in terms of its surplus and indicates how high the bidder should go before dropping out. Since $n \geq 3$, the prices at which some bidders drop out convey information (about the common value) to those who remain active. Suppose $k$ bidders have dropped out at bid levels $b_{1} \leq \ldots \leq b_{k}$. A bidder's (say $i$ 's) strategy is described by functions $B_{k}\left(S_{i} ; b_{1} \ldots b_{k}\right)$, which specify how high it must bid given that $k$ bidders have dropped out at levels $b_{1} \ldots b_{k}$ and given that its surplus is $S_{i}$. The $n$-tuple of strategies $(B(\cdot), \ldots, B(\cdot))$ with $B(\cdot))$ defined in Equation 3 , constitutes a symmetric equilibrium of the English auction.

$$
\begin{aligned}
B_{0}\left(x_{i 1}\right)= & E\left(v_{i 1}-c_{i 1} \mid S_{i 1}=x_{i 1}\right) \\
B_{k}\left(x_{i 1} ; b_{1} \ldots b_{k}\right)= & \frac{n-k}{n} E\left(v_{i 1} \mid S_{i 1}=x_{i 1}\right)+\frac{1}{n} \sum_{y=0}^{k-1} E\left(v_{i 1} \mid B_{y}\left(S_{i 1} ; b_{1}, \ldots, b_{y}\right)=b_{y+1}\right) \\
& -E\left(c_{i 1} \mid S_{i 1}=x_{i 1}\right)
\end{aligned}
$$

where $x_{i 1}$ is $i$ 's surplus. The intuition for Equation 3 is as follows. Given its surplus and the information conveyed in others' drop out levels, the highest a bidder is willing to go is given by the expected value of the object, assuming that all other active bidders have the same surplus. For instance, consider the bid function $B_{0}\left(S_{i 1}\right)$ which pertains to the case when no bidder has dropped out yet. If all other bidders were to drop out at level $B_{0}\left(S_{0}\right)$, then $i$ 's expected payoff $\left(e p=V_{1}-c_{i 1}-B_{0}\left(S_{0}\right)\right)$ would be:

$$
\begin{aligned}
e p & =S_{i 1}+\frac{n-1}{n} E\left(v \mid S=S_{0}\right)-B_{0}\left(S_{0}\right) \\
& =S_{i 1}+\frac{n-1}{n} E\left(v \mid S=S_{0}\right)-E\left(v-c \mid S=S_{0}\right) \\
& =S_{i 1}-S_{0}
\end{aligned}
$$


Using strategy $B_{0}, i$ remains active until it is indifferent between winning and quitting. Similar interpretations are given to $B_{k}$ for $k \geq 1$; the only difference is that these functions take into account the information conveyed in others' drop out levels.

Let $f_{1}^{n}$ denote the first order statistic of the surplus for the $n$ bidders and let $s_{1}^{n}$ denote the second order statistic. $f_{1}^{n}$ and $s_{1}^{n}$ are obtained from the distribution functions $Q_{1}$ and $G_{1}$. For the above equilibrium, it has been shown that the bidder with the highest surplus wins and the expected selling price (denoted $E\left(P_{w}\right)$ ) is [8]:

$$
E\left(P_{w}\right)=E\left(s_{1}^{n}\right)
$$

and the winner's expected profit (denoted $\left.E\left(\pi_{w}\right)\right)$ is:

$$
E\left(\pi_{w}\right)=E\left(f_{1}^{n}\right)-E\left(s_{1}^{n}\right)
$$

On the basis of the above equilibrium for a single object, we determine equilibrium for sequential auctions for the $m$ objects defined in Section 2 as follows.

Multiple objects. We will denote the first order statistic of the surplus for the $j$ th (for $j=1, \ldots, m)$ auction as $f_{j}^{n-j+1}$ and the second order statistic as $s_{j}^{n-j+1}$. Also, we denote a bidder's cumulative ex-ante expected profit from auctions $j$ to $m$ (where $1 \leq$ $j \leq m)$ as $\alpha_{j}$. Finally, we denote the winner's expected profit for the $j$ th auction as $E\left(\pi_{w j}\right)$. The following theorem characterises the equilibrium for $m>1$ objects.

Theorem 1. For $j=1, \ldots, m$, let $\beta_{j}=1 /(n-j+1)$ and let $\alpha_{j}$ be defined as:

$$
\alpha_{j}=\sum_{y=j}^{m}\left(\beta_{y} \times X_{y}\right)
$$

where $X_{y}=E\left(f_{y}^{n-y+1}\right)-E\left(s_{y}^{n-y+1}\right)+X_{y+1}$ for $y=1, \ldots, m$ and $X_{m+1}=0$. Then the $n$-tuple of strategies $(B(\cdot), \ldots, B(\cdot))$ with $B(\cdot)$ defined in Equation 7 constitutes an equilibrium for the $j$ th (for $j=1, \ldots,(m-1)$ ) auction at a stage where $k$ bidders have dropped out:

$$
\begin{aligned}
B_{0}^{j}\left(x_{i j}\right)= & E\left(v_{i j}-c_{i j} \mid S_{i j}=x_{i j}\right)-\alpha_{j+1} \\
B_{k}^{j}\left(x_{i j} ; b_{1}, \ldots, b_{k}\right)= & \frac{n-j+1-k}{n-z+1} E\left(v_{i j} \mid S_{i j}=x_{i j}\right)-E\left(c_{i j} \mid S_{i j}=x_{i j}\right) \\
& +\frac{1}{n-j+1} \sum_{y=0}^{k-1} E\left(v_{i j} \mid B_{y}\left(S_{i j} ; b_{1}, \ldots, b_{y}\right)=b_{y+1}\right) \\
& -\alpha_{j+1}
\end{aligned}
$$

For the last auction, the equilibrium is as given in Equation 3 with $n$ replaced with $(n-m+1)$.

For the above equilibrium, the winner for the $j$ th (for $j=1, \ldots, m$ ) auction is the bidder with the highest surplus for that auction (see proof of Theorem 2 in the appendix for details). 
Theorem 2. For the $j$ th (for $j=1, \ldots, m$ ) auction, the expected selling price (denoted $\left.E\left(P_{w j}\right)\right)$ is:

$$
\begin{aligned}
\forall_{j=1}^{m-1} E\left(P_{w j}\right) & =E\left(s_{j}^{n-j+1}\right)-\alpha_{j+1} \\
E\left(P_{w m}\right) & =E\left(s_{m}^{n-m+1}\right)
\end{aligned}
$$

Theorem 3. For the $j$ th (for $j=1, \ldots, m-1$ ) auction, the winner's expected profit (denoted $\left.E\left(\pi_{w j}\right)\right)$ is:

$$
E\left(\pi_{w j}\right)=E\left(f_{j}^{n-j+1}\right)-E\left(s_{j}^{n-j+1}\right)+\alpha_{j+1}
$$

and for the last auction, the winner's expected profit is:

$$
E\left(\pi_{w m}\right)=E\left(f_{m}^{n-m+1}\right)-E\left(s_{m}^{n-m+1}\right)
$$

In the following section, we use the above equilibrium to show how the expected selling price and the winner's expected profit vary from auction to auction.

\section{Selling price and winner's profit}

In Section 3, we determined equilibrium for the case where the distribution function for the value (cost) was different for different objects. In this section, our objective is to show that even if these distribution functions are identical across objects, the expected selling price is not the same for all objects. We present an example auction scenario which shows that in accordance with Ashenfelter's empirical result [1], the selling price for our model can decline in later auctions. Also, since the selling prices and the winner's profits depend on the number of bidders, we show that the equilibrium outcome in an agent-based setting (which has a relatively higher competition [16]), differs from that in an all human setting.

It must be noted that our objective here is not to provide a comprehensive study of how the expected selling price varies, but only to illustrate (with an example) that there exist cases where the variation predicted by our theoretical analysis accords with the experimental results of Ashenfelter [1].

Example auction scenario. This example in intended to show that:

- the selling price declines in later auctions (this result corresponds with Ashenfelter's empirical results [1]), and

- the winner's expected profit declines in later auctions.

In more detail, the setting for our analysis is as follows. The bidders' values are identically distributed across objects and so are their costs. The common values of all $m$ objects are drawn from a single distribution function. This function (say $Q: R_{+} \rightarrow$ $[0,1])$ is used to draw the common value of the $j$ th $(j=1, \ldots, m)$ object. Also, there is a single distribution function (say $G: R_{+} \rightarrow[0,1]$ ) for the cost of the $j$ th $(j=$ $1, \ldots, m)$ object. As before, each bidder receives a value signal (from $Q$ ) and a cost signal (from $G$ ) for an auction just before that auction begins. 


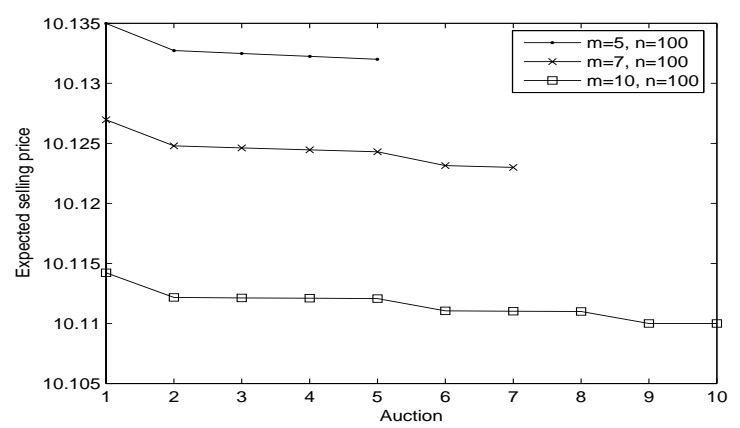

Fig. 1. A varying selling price in a series of auctions for the normal distribution.

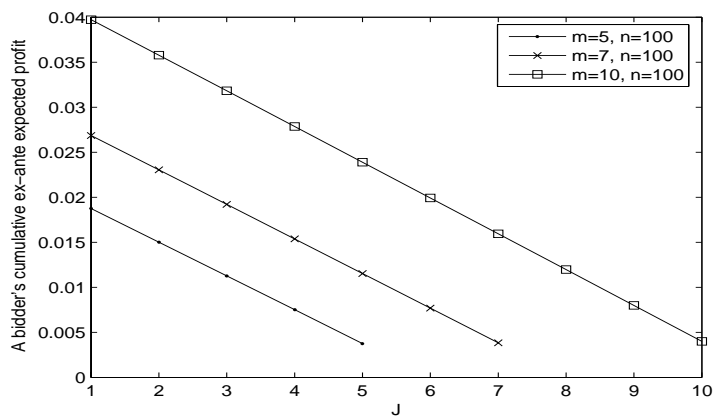

Fig. 2. A bidder's cumulative ex-ante expected profit $\left(\alpha_{j}\right)$ for the normal distribution.

Since there is a single distribution function for all objects, we drop the subscripts (for the order statistics) in Equations 8 and 9 and rewrite them as:

$$
E\left(P_{w j}\right)=E\left(s^{n-j+1}\right)-\alpha_{j+1}
$$

where $E\left(P_{w j}\right)$ denotes the expected selling price for the $j$ th auction. For the last auction, the selling price is:

$$
E\left(P_{w m}\right)=E\left(s^{n-m+1}\right)
$$

We determine the expected selling prices for the case where the values and costs are distributed normally. Recall that the normal distribution satisfies the log concavity assumption mentioned in Section 2. Both value and cost signals are distributed normally. The normal distribution is denoted $\mathcal{N}(\mu, \nu)$ where $\mu$ is the mean and $\nu$ is the variance. The mean and variance are taken as 10 and 0.5 respectively for the value signals, and 2 and 0.5 respectively for the cost signals. These two distributions for value and cost signals ensure that $c_{L}>0$ and $v_{L}>c_{H}$ for more than 99.8 percent of the popula- 


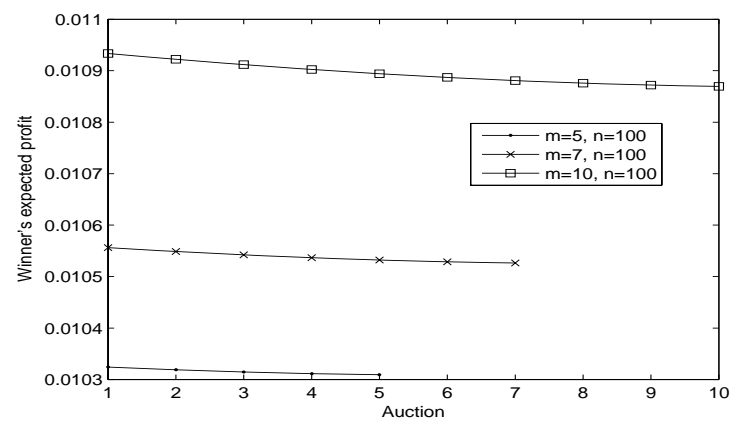

Fig. 3. The winner's expected profit for the normal distribution.

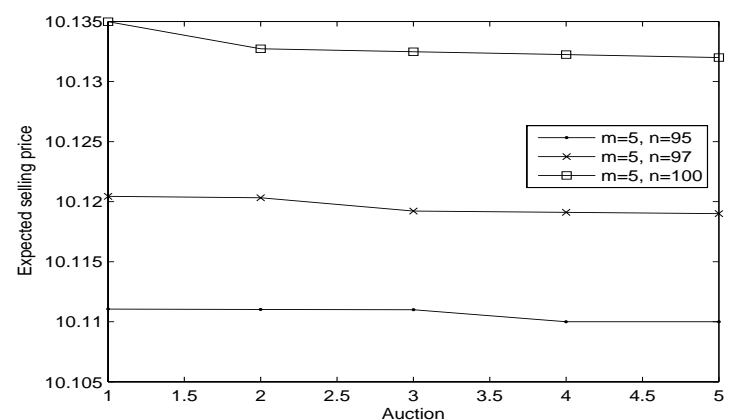

Fig. 4. The effect of competition on the expected selling price.

tion. Since the distribution for value is $\mathcal{N}(10,0.5)$ and that for cost is $\mathcal{N}(2,0.5)$, the distribution for the surplus (i.e., the difference of value and cost) is $\mathcal{N}(8,1)$ [5].

The first and second order statistics for the standard normal distribution (i.e., $\mathcal{N}(0,1)$ ) have been tabulated [9]. We use these tables to obtain the first and second order statistics for $\mathcal{N}(8,1)$ as follows. Since the distributions $\mathcal{N}(8,1)$ and $\mathcal{N}(0,1)$ differ only in their means, the first order statistics for $\mathcal{N}(8,1)$ is obtained by adding 8 to the first order statistics for $\mathcal{N}(0,1)$. Likewise, the second order statistics for $\mathcal{N}(8,1)$ is obtained by adding 8 to the second order statistics for $\mathcal{N}(0,1)$. Substituting these statistics in Equations 12 and 13 we get the expected selling price for each of the $m$ auctions.

The variation in the selling price (i.e., $\left.E\left(s^{n-j+1}\right)\right)-\alpha_{j+1}$ ) for different auctions is shown in Figure 1. As shown in the figure, the expected selling price decreases from one auction to the next. A bidder's ex-ante probability of winning the $j$ th auction (i.e., $\beta_{j}=$ $1 /(n-j+1))$ is increasing in $j$. A bidder's cumulative ex-ante expected profit from auctions $j$ to $m$ (i.e., $\alpha_{j}$ ) is shown in Figure 2. This profit decreases from one auction to the next. The winner's expected profit (i.e., $\left.E\left(\pi_{w j}\right)=E\left(f^{n-j+1}\right)-E\left(s^{n-j+1}\right)+\alpha_{j+1}\right)$ also drifts downward as shown in Figure 3. 


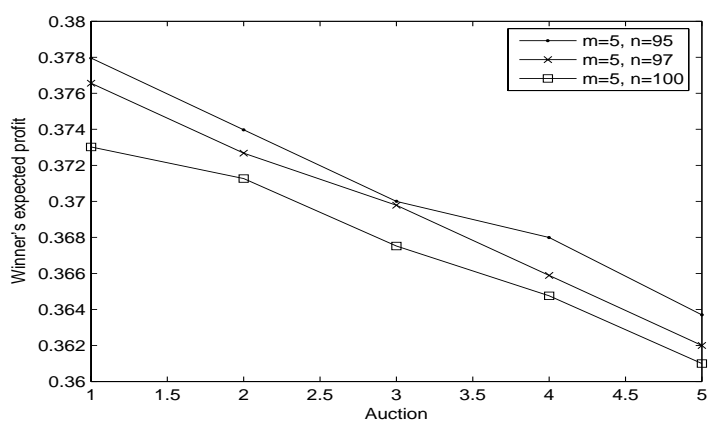

Fig. 5. The effect of competition on the winner's expected profit.

The effect of competition. In order to study the effect of competition, we fix the number of objects $(m)$ and vary the number of bidders $(n)$ for the example scenario described above. We know from Equations 12 and 13 that the expected selling price depends on the $E\left(s^{n-j+1}\right)$ and $\alpha_{j}$. For the normal distribution, both $E\left(s^{n-j+1}\right)$ and $\alpha_{j}$ decrease with $j$ [9]. Figure 4 is a plot of the expected selling price for different $n$. As seen in the figure, an increase in the number of bidders $(n)$ increases the selling price of each of the $m$ objects. Figure 5 is a plot of the winner's expected profit for different $n$. As seen in the figure, an increase in the number of bidders $(n)$ decreases the winner's profit for each of the $m$ objects. In summary, for the normal distribution, an increase in competition (i.e., increase in $n$ ) increases the selling price and decreases the winner's expected profit for each individual auction in a series. Since there is more competition in an agent-based setting than in a human setting [16], the expected selling prices are higher for the former setting relative to the latter, while the winner's expected profits are lower. In other words these two settings differ in terms of their equilibrium outcomes.

\section{Related Work}

Existing work has studied the dynamics of the selling price of objects for sequential auctions $[15,20,14,3]$. However, a key limitation of this work is that it focuses on objects that are either exclusively private value or exclusively common value. For instance, Ortega-Reichert [15] determined the equilibrium for sequential auctions for two private value objects using the first price rules. Weber [20] showed that in sequential auctions of identical objects with risk neutral bidders who hold independent private values, the expected selling price is the same for each auction. On the other hand, Milgrom and Weber [14] studied sequential auctions in an interdependent values model with affiliated $^{5}$ signals. They showed that expected selling prices have a tendency to drift upward.

\footnotetext{
${ }^{5}$ Affiliation is a form of positive correlation. Let $X_{1}, X_{2}, \ldots, X_{n}$ be a set of positively correlated random variables. Positive correlation roughly means that if a subset of $X_{i}$ s are large, then this makes it more likely that the remaining $X_{j}$ s are also large.
} 
This may be because earlier auctions release information about the values of objects and thereby reduce the winner's curse problem.

In contrast to the above theoretical results, there has been some evidence in realworld sequential auctions for identical objects - for art and wine auctions in particular that the prices tend to drift downward [1,13]. Because the theoretical models mentioned above predict either a stochastically constant or increasing price, this fact has been called the declining price anomaly. Mc Afee and Vincent [13] consider two identical private value objects and using the second price sealed bid rules, they show that the declining price anomaly cannot be explained even if the bidders are considered to be risk averse. Furthermore, Mc Afee and Vincent acknowledge that extending their two object model to a general $n$ object case is complex and they have not been able to come up with such a generalisation. We therefore adopted the model proposed by Bernhardt and Scoones for two private value objects [3] and generalised it to $n>2$ objects with both common and private values. Bernhardt and Scoones [3] showed that if the number of bidders for the first and second auctions are $n$ and $(n-1)$ respectively, the selling price can decline (by considering an example scenario). Our model is a generalization of [3] since we consider $n>2$ objects with both common and private values and show the decline in price for an example setting. Although the objects we consider have both common and private values, each bidder receives its signals for an auction just before the auction begins. In other words, during an auction, the information that bidders obtain about the others' value signals does not carry forward to subsequent auctions. Hence, as in the case of [3], our model too shows a decline in the selling price.

An important issue in the study of auctions with multi-dimensional signals is that of efficiency of auctions. In general, auctions with multi-dimensional signals have been shown to be inefficient [4]. Since our model involves two dimensional signals, we studied the efficiency property in [7]. This study shows that the efficiency of auctions in an agent-based setting is higher than that in an all human setting. This is because of the fact that an agent based setting leads to more competition than an all human setting [16].

\section{Conclusions and future work}

This paper has analyzed a model for sequential auctions for objects with private and common values in an uncertain information setting. We first determined equilibrium strategies for each auction in a sequence. Then we showed that even if the value and cost signals are distributed identically across objects, then in accordance with Ashenfelter's result [1], the expected selling price can decline in later auctions. Also, the winner's expected profit can decline in later auctions. We also showed that, due to differing competition, the equilibrium outcome in an agent-based setting differs from that in an all human setting.

There are many interesting directions for future work. First, our present focus was on determining how the expected selling price varies in a series of English auctions. However, in order to generalize our results, we intend to extend the analysis to other auction forms. Second, we studied the case where bidders received the cost and value signals for an object just before the auction. In future, we will extend the analysis to the 
case where the values and costs for the last $(m-1)$ objects can be determined from the signals for the first object (i.e., values and costs are perfectly correlated).

\section{References}

1. O. Ashenfelter. How auctions work for wine and art. Journal of Economic Perspectives, 3(221):23-36, 1989.

2. J. P. Benoit and V. Krishna. Multiple-object auctions with budget constrained bidders. Review of Economic Studies, 68:155-179, 2001.

3. D. Bernhardt and D. Scoones. A note on sequential auctions. The American Economic Review, 84(3):653-657, 1994.

4. P. Dasgupta and E. Maskin. Efficient auctions. Quarterly Journal of Economics, 115:341388, 2000.

5. M. Dwass. Probability and Statistics. W. A. Benjamin Inc., California, 1970.

6. W. Elmaghraby. The importance of ordering in sequential auctions. Management Science, 49(5):673-682, 2003.

7. S. S. Fatima, M. Wooldridge, and N. R. Jennings. Sequential auctions for objects with common and private values. In Proceedings of the Fourth International Conference on Autonomous Agents and Multi-Agent Systems, Utrecht, Netherlands (to appear), 2005.

8. J. K. Goeree and T. Offerman. Competitive bidding in auctions with private and common values. The Economic Journal, 113(489):598-613, 2003.

9. H. L. Harter. Expected values of normal order statistics. Biometrika, 48(1/2):151-165, 1961.

10. B. Katzman. A two stage sequential auction with multi-unit demands. Journal of Economic Theory, 86:77-99, 1999.

11. V. Krishna. Auction Theory. Academic Press, 2002.

12. J. Laffont. Game theory and empirical economics: The case of auction data. European Economic Review, 41:1-35, 1997.

13. R. P. McAfee and D. Vincent. The declining price anomaly. Journal of Economic Theory, 60:191-212, 1993.

14. P. Milgrom and R. J. Weber. A theory of auctions and competitive bidding II. In P. Klemperer, editor, The Economic Theory of Auctions. Edward Elgar, Cheltenham, U.K, 2000.

15. A. Ortega-Reichert. Models of competitive bidding under uncertainty. Technical Report 8 , Stanford University, 1968.

16. E. J. Pinker, A. Seidman, and Y. Vakrat. Managing online auctions: Current business and research issues. Management Science, 49(11):1457-1484, 2003.

17. C. Pitchik and A. Schotter. Perfect equilibria in budget-constrained sequential auctions: An experimental study. Rand Journal of Economics, 19:363-388, 1988.

18. T. Sandholm and S. Suri. BOB: Improved winner determination in combinatorial auctions and generalizations. Artificial Intelligence, 145:33-58, 2003.

19. W. Vickrey. Counterspeculation, auctions and competitive sealed tenders. Journal of Finance, 16:8-37, 1961.

20. R. J. Weber. Multiple-object auctions. In R. Engelbrecht-Wiggans, M. Shibik, and R. M. Stark, editors, Auctions, bidding, and contracting: Uses and theory, pages 165-191. New York University Press, 1983.

21. M. P. Wellman, W. E. Walsh, P. R. Wurman, and J. K. McKie-Mason. Auction protocols for decentralised scheduling. Games and Economic Behavior, 35:271-303, 2001.

22. R. E. Wiggans and C. M. Kahn. Multi-unit pay your-bid auctions with variable rewards. Games and Economic Behavior, 23:25-42, 1998. 
Appendix

\section{A Proof of Theorem 1}

Proof. We consider each of the $m$ auctions by reasoning backwards.

- $m$ th auction. To begin, consider the $m$ th auction for which there are $(n-m+1)$ bidders. Since this is the last auction, an agent's bidding behaviour is the same as that for the single object case. Hence, the equilibrium for this auction is the same as that in Equation 3 with $n$ replaced with $(n-m+1)$. For $j=1, \ldots, m$, let $\alpha_{i j}$ denote an agent's cumulative ex-ante expected profit from auctions $j$ to $m$. Recall that although the bidders know the distribution (from which the cost and value signals are drawn) before the first auction begins, they draw the signals for the $j$ th auction only after the $(j-1)$ earlier auctions end. Since $\alpha_{i j}$ is the ex-ante expected profit (i.e., it is computed before the bidders draw their signals for the $j$ th auction), it is the same for all participating bidders. Thus, we will simplify notation by dropping the subscript $i$ and denote $\alpha_{i j}$ simply as $\alpha_{j}$ We know from Equation 5 that:

$$
\alpha_{m}=\frac{1}{n-m+1}\left(E\left(f_{m}^{n-m+1}\right)-E\left(s_{m}^{n-m+1}\right)\right)
$$

This is because all the $(n-m+1)$ agents that participate in the $m$ th auction have ex-ante identical chances of winning it. Note that the right hand side of Equation 14 does not depend on $i$. In other words, since bidders receive their signals for the $m$ th auction after the $(m-1)$ th auction, the ex-ante expected profit for the $m$ th auction (before the $(m-1)$ th auction ends) is the same for all the $(n-m+1)$ bidders.

- $(m-1)$ th auction. Consider the $(m-1)$ th auction. During this auction, a bidder bids $b$ if $\left(V_{m-1}-c_{m-1}-b \geq \alpha_{m}\right)$ or $\left(b \leq V_{m-1}-c_{m-1}-\alpha_{m}\right)$. Hence, a symmetric equilibrium for the $(m-1)$ th auction is obtained by substituting $j=m-1$ in Equation 7. We know from Equation 4, that the expected selling price for the single object case is the second order statistic of the surplus. The difference between the equilibrium bids for the single object case and the $(m-1)$ th auction of the $m$ objects case is $\alpha_{m}$ (see Equations 3 and 7). Hence, the expected selling price for the $(m-1)$ th auction is $E\left(s_{m-1}^{n-m+2}\right)-\alpha_{m}$. This implies that the winner's expected profit for the $(m-1)$ th auction is:

$$
E\left(\pi_{w(m-1)}\right)=E\left(f_{m-1}^{n-m+2}\right)-E\left(s_{m-1}^{n-m+2}\right)+\alpha_{m}
$$

- First $(m-2)$ auctions. Consider the $j$ th auction where $1 \leq j \leq m-2$. We now find $\alpha_{1}, \ldots, \alpha_{m-1}$. Since the number of bidders for the $j$ th auction is $(n-j+1)$ and each bidder has ex-ante equal chances of winning, the probability that a bidder wins the $j$ th (for $j=1, \ldots, m$ ) auction (denoted $\beta_{j}$ ) is:

$$
\beta_{j}=1 /(n-j+1)
$$


This implies that, for $j=1, \ldots, m, \alpha_{j}$ is:

$$
\alpha_{j}=\beta_{j} E\left(\pi_{w j}\right)+\alpha_{j+1}
$$

Generalising Equation 15 to the first $(m-1)$ auctions, we get the winner's expected profit $\left(E\left(\pi_{w j}\right)\right)$ as:

$$
E\left(\pi_{w j}\right)=E\left(f_{j}^{n-j+1}\right)-E\left(s_{j}^{n-j+1}\right)+\alpha_{j+1}
$$

Consequently, a bidder's optimal bid for the $j$ th auction is obtained by discounting the single object equilibrium bid by $\alpha_{j+1}$. Hence, we get the equilibrium bids in Equation 7.

\section{B Proof of Theorem 2}

Proof. It is important to note that for the $j$ th $(j=1, \ldots, m)$ auction, the bids in Theorem 1 are similar to those in Equation 3 (for the single object case), except that each bid in the former case is obtained from the corresponding bid in the latter by shifting the latter by the constant $\alpha_{j+1}$. Since $\alpha_{j+1}$ is the same for all participating bidders, the relative positions of bidders for each of the $m$ auctions remains the same as that for the corresponding single object case. Hence, for each of the $m$ auctions, the winner is the bidder with the highest surplus for that auction. Consequently, the expected selling price for the $j$ th (for $j=1, \ldots, m-1$ ) auction is $E\left(P_{w j}\right)=E\left(s_{j}^{n-j+1}\right)-\alpha_{j+1}$. For the last auction (which is similar to a single object auction), the selling price is: $E\left(P_{w m}\right)=E\left(s_{m}^{n-m+1}\right)$.

\section{Proof of Theorem 3}

Proof. The $m$ th auction is identical to the single object case. Hence, the expected profit for this auction is

$$
E\left(\pi_{w m}\right)=E\left(f_{m}^{n-m+1}\right)-E\left(s_{m}^{n-m+1}\right)
$$

Since the difference between the expected selling price for the single object case and the $j$ th (for $j=1, \ldots, m-1$ ) auction of the $m$ objects case is $\alpha_{j+1}$, the winner's expected profit for the $j$ th auction is:

$$
E\left(\pi_{w j}\right)=E\left(f_{j}^{n-j+1}\right)-E\left(s_{j}^{n-j+1}\right)+\alpha_{j+1}
$$

